\title{
The Effect of Trading Activity and Holdings Market Capitalization on Portfolio Performance
}

\author{
Pei Lin Deng ${ }^{1}$ \\ ${ }^{1}$ Sichuan College of Architectural Technology, Deyang, Sichuan Province, China \\ Correspondence: Pei Lin Deng, Department of Economics, Sichuan College of Architectural Technology, No. 4 \\ Jia Ling Jiang Xi Road, Deyang, Sichuan Province, China. Tel: 86-838-265-2059. E-mail: dengpl@ scac.edu.cn
}

Received: May 19, 2018

Accepted: June 19, 2018

Online Published: June 28, 2018

doi:10.5539/ijef.v10n8p

URL: https://doi.org/10.5539/ijef.v10n8p18

\begin{abstract}
This paper augments a growing body of empirical literature on the turnover-return relationship of stock portfolios. From quarterly data over the recent decade, mutual funds that focus on smaller cap stocks are found to pay a greater performance penalty for active trading compared to those that focus on larger cap stocks. On average, managers in every fund focus type make investment decisions that benefit gross returns. However, detriment from excessive trading arises due to transaction costs. They are especially magnified for mutual funds that focus on smaller cap stocks. Findings herein also support previous studies that show fund managers execute lower-quality trades with tenure. The presented effect modification of trading activity by holdings focus type and manager tenure may be leveraged to refine portfolio investment strategies.
\end{abstract}

Keywords: trading activity, portfolio turnover, mutual fund returns, transaction costs, market capitalization

\section{Introduction}

Carhart (1997) documented the negative impact of portfolio turnover on returns for mutual funds. Barber and Odean (2000) demonstrated the same phenomenon for individual investors. The current work investigates how turnover impacts returns for portfolios that differ in their holdings concentration. Specifically, effect sizes are assessed for mutual funds that focus on stocks of different market capitalization: large cap, mid cap, small cap, or micro cap.

Naïve preliminary regressions of mutual fund excess net returns on fund turnover rate and Fama-French market factors produce a surprising result. Coefficient estimates suggest that active trading is detrimental to the excess returns of larger cap focused mutual funds and beneficial to the excess returns of smaller cap focused mutual funds. This seems counterintuitive because there is much less available information to accurately value smaller cap stocks. Accordingly, the market makers that handle these securities try to protect themselves against information asymmetry and adverse selection by raising the costs of trading (Glosten \& Milgrom, 1985; Sadka \& Scherbina, 2007). Therefore smaller cap mutual funds would be expected to lose more from trading, simply due to the illiquidity of their holdings.

These initial findings are not robust to other methods of evaluating turnover impact. With respect to own-benchmark abnormal returns (Barber \& Odean, 2000), smaller cap funds tend to have lower annual net returns compared to what would have been earned following a buy-and-hold strategy for a year. Even so, the average own-benchmark abnormal return is negative for every type of fund. The negative magnitude decreases with increasing market cap focus. A complementary result is derived from regressions of mutual fund excess gross return on fund turnover rate and Fama-French market factors. Under this analysis, portfolio turnover exhibits a positive effect on excess gross return for every type of fund, which implies that fund managers on average do take profitable investment positions and that negative own-benchmark abnormal returns are mainly due to transaction costs. As expected, such costs are highest for smaller cap stocks, so it is sensible that micro cap funds have the poorest-performing abnormal returns.

Inconsistency among results from the multiple analytical approaches above can be resolved by adding a turnover-manager interactive variable to each regression. Inspiration for this variable construction comes from Jin and Scherbina (2011), who reported that managers progressively hold on to more losers as they work longer at the same fund. The coefficient on the initial turnover term becomes positive and significant, and this new 
interactive term carries a negative coefficient. Therefore its original omission imposed a downward bias on the turnover term's marginal impact. The bias was large enough in magnitude to make the coefficient negative in the large cap and mid cap regressions.

Trading activity exhibits a non-uniform effect on portfolio return that differs by holdings market capitalization focus and manager tenure. Investment fund leadership may utilize the following framework and discussion to re-calibrate profit generating strategies.

\section{Method}

\subsection{Mutual Funds Quarterly Data}

Quarterly net returns, turnover rate, expense ratio, and manager tenure were obtained for every large cap, mid cap, small cap, and micro cap focused mutual fund in the Center for Research in Security Prices (CRSP) Survivor-Bias-Free U.S. Mutual Fund Database. Quarterly turnover rate is defined as the beginning-of-quarter market value of shares purchased in the previous quarter (or sold in the in the current quarter) divided by the total beginning-of-quarter market value of shares. Quarterly expense ratio is defined as annual operating expenses divided by the value of assets under management, further divided by 4 . Manager tenure is defined as the difference between observation date and most recent manager-change date. Market capitalization focus categories were identified based on Lipper Classification Name. All balanced-type funds were omitted from this study. The time period of interest was March 2001 through December 2010. In total, quarterly observations were available for 10016 mutual funds: 3362 focused in large cap stocks, 3301 focused in mid cap stocks, 3237 focused in small cap stocks, and 116 focused in micro cap stocks.

The year 2001 was chosen as a lower bound because CRSP's mutual fund holdings data only go back to 2001 . Holdings were necessary to evaluate fund performance in the absence of fees (i.e. gross returns). Quarterly gross return of each fund was calculated as the value-weighted mean gross return of the securities held by the fund. The marginal impact of turnover rate could then be assessed in both the presence and absence of transaction costs. The year 2010 was chosen as an upper bound because holdings information is less complete in more recent years such that profound non-random missing data may compromise analyses.

\subsection{Measuring the Marginal Impact of Turnover on Fund Performance}

Mutual fund quarterly excess returns were regressed on turnover rate in the current and previous quarter, Fama-French factors, expense ratio in the current and previous quarter, NYSE/AMEX/NASDAQ decile-1 capitalization quarterly excess returns, and the number of years from the most recent manager change. This was performed for each of the four different fund focus categories:

$$
\begin{aligned}
\left(R_{i t}-R_{f t}\right)= & \alpha_{i}+\tau_{i} \cdot \text { TURN }_{i t}+\tau_{i}{ }^{\prime} \cdot \operatorname{TURN}_{i t-1}+\beta_{i} \cdot\left(R_{m t}-R_{f t}\right)+s_{i} \cdot S M B_{t}+h_{i} \cdot H M L_{t} \\
& +\gamma_{i} \cdot \operatorname{EXP}_{i t}+\gamma_{i}{ }^{\prime} \cdot \operatorname{EXP}_{i t-1}+\delta_{i} \cdot \text { MICRO }_{t}+\zeta_{i} \cdot M G R Y R S_{i t}+\epsilon_{i t}
\end{aligned}
$$

The subscript $i$ denotes one of four fund focus categories; $t$ denotes time in quarters. $R_{i t}$ is the return for a category $i$ mutual fund during quarter $t$, modeled separately as net return and as gross return. $R_{f t}$ is the risk-free return during quarter $t . R_{i t}-R_{f t}$ is the quarterly excess return. $R_{m t}$ is the quarterly average market return. $R_{m t}-R_{f t}$ is the quarterly market excess return. $T U R N_{i t}$ is the quarterly turnover rate. $S M B_{t}$ is the quarterly return on a value-weighted portfolio of small stocks minus the quarterly return on a value-weighted portfolio of large stocks. $H M L_{t}$ is the quarterly return on a value-weighted portfolio of high book-to-market stocks minus the quarterly return on a value-weighted portfolio of low book-to-market stocks. EXP $P_{i t}$ is the quarterly expense ratio. $M I C R O_{t}$ is the quarterly excess return of NYSE/AMEX/NASDAQ stocks in the smallest capitalization decile. MGRYRS is the number of years since the most recent manager change. The intercept is $\alpha_{i}$ and the error term is $\epsilon_{i t}$. Inclusion of lagged turnover and expense variables considers the possibility that turnover in the present may be driven by prior returns (Chevalier and Ellison, 1997).

\subsection{Measuring the Total Annual Impact of Turnover on Fund Performance}

An average annual own-benchmark abnormal return was computed for each type of fund following the method of Barber and Odean (2000). For every mutual fund, the one-year future gross return of the previous year's portfolio, less the expense ratio, was subtracted from the net annual return of the current year. In other words, the return earned by each fund had it simply held its beginning-of-year portfolio for an entire year and paid usual operating costs was used as a benchmark. Since transaction costs are not factored into the expense ratio, the abnormal return obtained from differencing out the benchmark return represents the overall impact of trading, including transaction costs, over the course of a year. 


\section{Results}

Table 1 presents descriptive statistics for all of the mutual funds in this study. Not surprisingly, funds that focus on smaller cap stocks have greater gross and net returns than those focusing on larger cap stocks. The standard deviation of returns trends upward with decreasing market cap size. The distributions of market value and number of shares held are all highly upward-skewed. In every fund focus category, the mean market value and number of shares held exceed the 75th percentile mark. The largest fund in this sample is a mid cap fund, with total market value of $\$ 22.2$ billion. The fund holding the most number of shares is a small cap fund, with 3.8 billion shares.

At the average, micro cap funds hold both the greatest market value and the most number of shares. Average turnover rate and expense ratio are otherwise similar across these fund focus categories. Large, mid, and small cap funds also have similar average manager tenure, at around 5 years. Managers at micro cap funds, however, change much more frequently. Large, mid, and small cap fund managers can hold their positions for as long as two and a half decades, while the tenure of micro cap fund managers in this sample tops out at 8 years.

Table 1. Descriptive statistics of mutual fund returns, turnover, expenses, holdings, and management change

\begin{tabular}{|c|c|c|c|c|c|c|c|}
\hline & Mean & 25 th $\%$ tile & Median & 75th \%tile & St. Dev. & Min. & Max. \\
\hline \multicolumn{8}{|c|}{ Panel A: Large Cap (3362 Funds) } \\
\hline Quarterly Net Return & 0.0043 & -0.0489 & 0.0188 & 0.0656 & 0.0989 & -0.4470 & 0.5156 \\
\hline Quarterly Gross Return & 0.0984 & 0.0308 & 0.0859 & 0.1396 & 0.1407 & -0.3839 & 1.9030 \\
\hline Quarterly Turnover Rate & 0.93 & 0.39 & 0.68 & 1.10 & 1.22 & 0.01 & 20.18 \\
\hline Quarterly Expense Ratio & 0.0033 & 0.0024 & 0.0031 & 0.0712 & 0.0215 & 0.0000 & 0.1560 \\
\hline Market Value & $\$ 150 \mathrm{~m}$ & $\$ 1.34 \mathrm{~m}$ & $\$ 7.68 \mathrm{~m}$ & $\$ 49.5 \mathrm{~m}$ & $\$ 530 \mathrm{~m}$ & $\$ 125.49$ & $\$ 13.7 \mathrm{~b}$ \\
\hline Number of Shares & $5.0 \mathrm{~m}$ & 35000 & 205208 & $1.6 \mathrm{~m}$ & 190343 & 3 & $422 \mathrm{~m}$ \\
\hline Manager Tenure (in years) & 5.1 & 2.7 & 4.2 & 6.6 & 3.4 & 0.4 & 26.7 \\
\hline \multicolumn{8}{|c|}{ Panel B: Mid Cap (3301 Funds) } \\
\hline Quarterly Net Return & 0.0130 & -0.0494 & 0.0314 & 0.0804 & 0.1162 & -0.6648 & 0.8171 \\
\hline Quarterly Gross Return & 0.1175 & 0.0479 & 0.1193 & 0.1754 & 0.1587 & -0.6311 & 2.4759 \\
\hline Quarterly Turnover Rate & 1.08 & 0.46 & 0.78 & 1.31 & 1.66 & 0.00 & 37.27 \\
\hline Quarterly Expense Ratio & 0.0560 & 0.0400 & 0.0524 & 0.0740 & 0.0228 & 0.0000 & 0.1996 \\
\hline Market Value & $\$ 227 \mathrm{~m}$ & $\$ 1.20 \mathrm{~m}$ & $\$ 6.40 \mathrm{~m}$ & $\$ 61.9 \mathrm{~m}$ & $\$ 1.01 \mathrm{~b}$ & $\$ 19.15$ & $\$ 22.2 \mathrm{~b}$ \\
\hline Number of Shares & $10.4 \mathrm{~m}$ & 39175 & 228387 & $2.9 \mathrm{~m}$ & $44.0 \mathrm{~m}$ & 1 & $957 \mathrm{~m}$ \\
\hline Manager Tenure (in years) & 5.2 & 2.7 & 4.4 & 7.2 & 3.2 & 0.2 & 27.0 \\
\hline \multicolumn{8}{|c|}{ Panel C: Small Cap (3237 Funds) } \\
\hline Quarterly Net Return & 0.0158 & -0.0518 & 0.0237 & 0.0883 & 0.1244 & -0.6599 & 8.1478 \\
\hline Quarterly Gross Return & 0.1404 & 0.0502 & 0.1362 & 0.2019 & 0.1965 & -0.6239 & 9.2188 \\
\hline Quarterly Turnover Rate & 0.86 & 0.43 & 0.70 & 1.14 & 0.62 & 0.01 & 6.00 \\
\hline Quarterly Expense Ratio & 0.0612 & 0.0452 & 0.0572 & 0.0784 & 0.0224 & 0.0060 & 0.2388 \\
\hline Market Value & $\$ 141 \mathrm{~m}$ & $\$ 73,260$ & $\$ 3.85 \mathrm{~m}$ & $\$ 56.8 \mathrm{~m}$ & $\$ 510 \mathrm{~m}$ & $\$ 35.00$ & $\$ 8.38 \mathrm{~b}$ \\
\hline Number of Shares & $13.0 \mathrm{~m}$ & 36700 & 200000 & $3.4 \mathrm{~m}$ & $114 \mathrm{~m}$ & 15 & $3.80 \mathrm{~b}$ \\
\hline Manager Tenure (in years) & 5.2 & 2.7 & 4.2 & 7.2 & 3.2 & 0.2 & 24.2 \\
\hline \multicolumn{8}{|c|}{ Panel D: Micro Cap (116 Funds) } \\
\hline Quarterly Net Return & 0.0240 & -0.4077 & 0.0463 & 0.5617 & 0.4369 & -0.7699 & 1.4487 \\
\hline Quarterly Gross Return & 0.1428 & 0.0456 & 0.1377 & 0.6147 & 0.1511 & -0.7073 & 5.4840 \\
\hline Quarterly Turnover Rate & 0.88 & 0.57 & 1.00 & 1.21 & 0.34 & 0.12 & 1.27 \\
\hline Quarterly Expense Ratio & 0.0620 & 0.0452 & 0.0596 & 0.0896 & 0.0220 & 0.0216 & 0.3072 \\
\hline Market Value & $\$ 282 \mathrm{~m}$ & $\$ 2.21 \mathrm{~m}$ & $\$ 136 \mathrm{~m}$ & $\$ 177 \mathrm{~m}$ & $\$ 673 \mathrm{~m}$ & $\$ 373,272$ & $\$ 3.56 \mathrm{~b}$ \\
\hline Number of Shares & $28.1 \mathrm{~m}$ & 163082 & $11.7 \mathrm{~m}$ & $15.8 \mathrm{~m}$ & $66.4 \mathrm{~m}$ & 31778 & $315 \mathrm{~m}$ \\
\hline Manager Tenure (in years) & 3.4 & 2.4 & 4.2 & 6.7 & 2.8 & 0.2 & 8.2 \\
\hline
\end{tabular}

\subsection{Marginal Impact of Turnover on Fund Performance}

Table 2 presents the regression results for Equation 1 using net returns. Table 3 is analogous to Table 2 for gross returns. In Table 2, the benefit of active trading appears to increase as the market cap of mutual fund holdings decreases. Portfolio turnover is negatively associated with the excess net returns of large cap and mid cap funds 
for all of the specifications in Panels A and B. On the other hand, the average excess net return of small cap and micro cap funds increases with turnover. The relationship is especially pronounced for micro cap funds. On average, a $1 \%$ increase in quarterly turnover rate increases the excess net return of micro cap funds by just over 5 basis points in the regression with only Fama-French controls. However, this positive impact is almost entirely explained away through inclusion of the smallest-cap-stocks excess return term. Nevertheless, even with this control, turnover exhibits a negative and significant association with excess net returns of large cap funds.

Table 2. Regression of mutual fund excess net returns on turnover rate and other controls

\begin{tabular}{|c|c|c|c|c|c|c|}
\hline \multicolumn{7}{|c|}{ Panel A: Large Cap (3362 Funds) } \\
\hline & $(1)$ & $(2)$ & (3) & (4) & $(5)$ & $(6)$ \\
\hline$\alpha_{i}$ & $\begin{array}{l}0.00627 * * * \\
\left(<1 \times 10^{-12}\right)\end{array}$ & $\begin{array}{l}0.00339 * * * \\
\left(<1 \times 10^{-12}\right)\end{array}$ & $\begin{array}{l}0.00569^{* * * *} \\
\left(<1 \times 10^{-12}\right)\end{array}$ & $\begin{array}{l}0.00625^{* * *} \\
\left(<1 \times 10^{-12}\right)\end{array}$ & $\begin{array}{c}0.00276^{* * *} \\
\left(2.19 \times 10^{-5}\right)\end{array}$ & $\begin{array}{c}0.00220 * * * \\
(0.000760)\end{array}$ \\
\hline$T U R N_{i t}$ & $\begin{array}{c}-6.48 \times 10^{-5 * * *} \\
(0.00242)\end{array}$ & $\begin{array}{c}-7.05 \times 10^{-5} * * * \\
(0.000962)\end{array}$ & $\begin{array}{c}-6.20 \times 10^{-5} * * * \\
(0.00365)\end{array}$ & $\begin{array}{c}-0.000140^{* * *} \\
\left(1.74 \times 10^{-6}\right)\end{array}$ & $\begin{array}{c}-0.000144^{* * *} \\
\left(8.32 \times 10^{-7}\right)\end{array}$ & $\begin{array}{c}-0.000244 * * * \\
\left(3.48 \times 10^{-5}\right)\end{array}$ \\
\hline$T U R N_{i t-1}$ & & & & & & $\begin{array}{c}0.000124 \\
(0.362)\end{array}$ \\
\hline$R_{m t}-R_{f t}$ & $\begin{array}{c}0.961 * * * \\
\left(<1 \times 10^{-12}\right)\end{array}$ & $\begin{array}{c}0.961 * * * \\
\left(<1 \times 10^{-12}\right)\end{array}$ & $\begin{array}{c}0.978 * * * \\
\left(<1 \times 10^{-12}\right)\end{array}$ & $\begin{array}{c}0.965^{* * * *} \\
\left(<1 \times 10^{-12}\right)\end{array}$ & $\begin{array}{c}0.981^{* * *} \\
\left(<1 \times 10^{-12}\right)\end{array}$ & $\begin{array}{c}0.987 * * * \\
\left(<1 \times 10^{-12}\right)\end{array}$ \\
\hline$S M B_{t}$ & $\begin{array}{l}-0.0809^{* * *} \\
\left(<1 \times 10^{-12}\right)\end{array}$ & $\begin{array}{l}-0.0805^{* * * *} \\
\left(<1 \times 10^{-12}\right)\end{array}$ & $\begin{array}{l}-0.0658^{* * *} \\
\left(<1 \times 10^{-12}\right)\end{array}$ & $\begin{array}{l}-0.0735^{* * * *} \\
\left(<1 \times 10^{-12}\right)\end{array}$ & $\begin{array}{l}-0.0587 * * * \\
\left(<1 \times 10^{-12}\right)\end{array}$ & $\begin{array}{l}-0.0532^{* * *} \\
\left(<1 \times 10^{-12}\right)\end{array}$ \\
\hline$H M L_{t}$ & $\begin{array}{l}-0.0374^{* * *} \\
\left(<1 \times 10^{-12}\right)\end{array}$ & $\begin{array}{l}-0.0373^{* * *} \\
\left(<1 \times 10^{-12}\right)\end{array}$ & $\begin{array}{l}-0.0393^{* * *} \\
\left(<1 \times 10^{-12}\right)\end{array}$ & $\begin{array}{l}-0.0438^{* * * *} \\
\left(<1 \times 10^{-12}\right)\end{array}$ & $\begin{array}{l}-0.0456^{* * *} \\
\left(<1 \times 10^{-12}\right)\end{array}$ & $\begin{array}{l}-0.0483^{* * *} \\
\left(<1 \times 10^{-12}\right)\end{array}$ \\
\hline$E X P_{i t}$ & & $\begin{array}{l}-0.835^{* * *} \\
\left(<1 \times 10^{-12}\right)\end{array}$ & & & $\begin{array}{c}-0.816^{* * *} \\
\left(2.01 \times 10^{-12}\right)\end{array}$ & $\begin{array}{l}-1.04 * * * \\
(0.00392)\end{array}$ \\
\hline$E X P_{i t-1}$ & & & & & & $\begin{array}{c}3.281 \\
(0.234)\end{array}$ \\
\hline $\mathrm{MICRO}_{t}$ & & & $\begin{array}{l}-0.0356^{* * *} \\
\left(<1 \times 10^{-12}\right)\end{array}$ & & $\begin{array}{l}-0.0488^{* * *} \\
\left(<1 \times 10^{-12}\right)\end{array}$ & $\begin{array}{l}-0.0173^{* * *} \\
\left(<1 \times 10^{-12}\right)\end{array}$ \\
\hline$M_{G R Y R S_{i t}}$ & & & & $\begin{array}{c}-5.70 \times 10^{-5} \\
(0.398)\end{array}$ & $\begin{array}{c}-7.17 \times 10^{-5} \\
(0.288)\end{array}$ & $\begin{array}{c}-9.72 \times 10^{-5} \\
(0.150)\end{array}$ \\
\hline Obs. & 36006 & 36006 & 36006 & 22691 & 22691 & 20946 \\
\hline$R^{2}$ & 0.896 & 0.896 & 0.896 & 0.893 & 0.893 & 0.897 \\
\hline \multicolumn{7}{|c|}{ Panel B: Mid Cap (3301 Funds) } \\
\hline & $(1)$ & $(2)$ & (3) & (4) & (5) & (6) \\
\hline$\alpha_{i}$ & $\begin{array}{l}0.00212^{* * * *} \\
\left(<1 \times 10^{-12}\right)\end{array}$ & $\begin{array}{l}0.00212^{* * * *} \\
\left(<1 \times 10^{-12}\right)\end{array}$ & $\begin{array}{l}0.00357^{* * * *} \\
\left(<1 \times 10^{-12}\right)\end{array}$ & $\begin{array}{c}0.00344 * * * \\
\left(7.71 \times 10^{-11}\right)\end{array}$ & $\begin{array}{l}0.00544^{* * * *} \\
\left(<1 \times 10^{-12}\right)\end{array}$ & $\begin{array}{l}0.00574 * * * \\
\left(<1 \times 10^{-12}\right)\end{array}$ \\
\hline$T U R N_{i t}$ & $\begin{array}{c}-3.94 \times 10^{-5} \\
(0.436)\end{array}$ & $\begin{array}{c}-3.94 \times 10^{-5} \\
(0.436)\end{array}$ & $\begin{array}{c}-4.34 \times 10^{-5} \\
(0.391)\end{array}$ & $\begin{array}{c}-0.000134 \\
(0.203)\end{array}$ & $\begin{array}{c}-0.000129 \\
(0.221)\end{array}$ & $\begin{array}{c}-0.000137^{* *} \\
(0.0427)\end{array}$ \\
\hline$T U R N_{i t-1}$ & & & & & & $\begin{array}{c}0.000188 \\
(0.593)\end{array}$ \\
\hline$R_{m t}-R_{f t}$ & $\begin{array}{c}1.040^{* * * *} \\
\left(<1 \times 10^{-12}\right)\end{array}$ & $\begin{array}{c}1.040^{* * * *} \\
\left(<1 \times 10^{-12}\right)\end{array}$ & $\begin{array}{c}0.996^{* * * *} \\
\left(<1 \times 10^{-12}\right)\end{array}$ & $\begin{array}{c}1.047 * * * \\
\left(<1 \times 10^{-12}\right)\end{array}$ & $\begin{array}{c}0.991 * * * \\
\left(<1 \times 10^{-12}\right)\end{array}$ & $\begin{array}{c}0.962^{* * * *} \\
\left(<1 \times 10^{-12}\right)\end{array}$ \\
\hline$S M B_{t}$ & $\begin{array}{c}0.204^{* * *} \\
\left(<1 \times 10^{-12}\right)\end{array}$ & $\begin{array}{c}0.204 * * * \\
\left(<1 \times 10^{-12}\right)\end{array}$ & $\begin{array}{c}0.163 * * * \\
\left(<1 \times 10^{-12}\right)\end{array}$ & $\begin{array}{c}0.228 * * * \\
\left(<1 \times 10^{-12}\right)\end{array}$ & $\begin{array}{c}0.171^{* * * *} \\
\left(<1 \times 10^{-12}\right)\end{array}$ & $\begin{array}{c}0.161^{* * *} \\
\left(<1 \times 10^{-12}\right)\end{array}$ \\
\hline$H M L_{t}$ & $\begin{array}{l}-0.111^{* * *} \\
\left(<1 \times 10^{-12}\right)\end{array}$ & $\begin{array}{l}-0.111^{* * *} \\
\left(<1 \times 10^{-12}\right)\end{array}$ & $\begin{array}{l}-0.107^{* * *} \\
\left(<1 \times 10^{-12}\right)\end{array}$ & $\begin{array}{l}-0.136^{* * *} \\
\left(<1 \times 10^{-12}\right)\end{array}$ & $\begin{array}{l}-0.134 * * * \\
\left(<1 \times 10^{-12}\right)\end{array}$ & $\begin{array}{l}-0.113^{* * *} \\
\left(<1 \times 10^{-12}\right)\end{array}$ \\
\hline$E X P_{i t}$ & & $\begin{array}{c}-0.000581 \\
(0.347)\end{array}$ & & & $\begin{array}{c}-0.000574 \\
(0.374)\end{array}$ & $\begin{array}{c}0.000421 \\
(0.735)\end{array}$ \\
\hline$E X P_{i t-1}$ & & & & & & $\begin{array}{c}-0.00131 \\
(0.364)\end{array}$ \\
\hline$M_{I C R O}$ & & & $\begin{array}{l}-0.0140^{* * *} \\
\left(<1 \times 10^{-12}\right)\end{array}$ & & $\begin{array}{l}-0.0142 * * * \\
\left(3.62 \times 10^{-10}\right)\end{array}$ & $\begin{array}{c}0.0634 * * * \\
\left(<1 \times 10^{-12}\right)\end{array}$ \\
\hline$M G R Y R S_{i t}$ & & & & $\begin{array}{c}-0.000317^{* * *} \\
(0.00106)\end{array}$ & $\begin{array}{c}-0.000324 * * * \\
(0.000767)\end{array}$ & $\begin{array}{c}0.000273^{* * * *} \\
(0.00441)\end{array}$ \\
\hline Obs. & 37396 & 37396 & 37396 & 24734 & 24734 & 23092 \\
\hline$R^{2}$ & 0.844 & 0.844 & 0.844 & 0.827 & 0.828 & 0.831 \\
\hline
\end{tabular}




\begin{tabular}{|c|c|c|c|c|c|c|}
\hline \multicolumn{7}{|c|}{ Panel C: Small Cap (3237 Funds) } \\
\hline & $(1)$ & $(2)$ & (3) & $(4)$ & $(5)$ & $(6)$ \\
\hline$\alpha_{i}$ & $\begin{array}{l}0.00516^{* * * *} \\
\left(<1 \times 10^{-12}\right)\end{array}$ & $\begin{array}{l}0.00516^{* * * *} \\
\left(<1 \times 10^{-12}\right)\end{array}$ & $\begin{array}{l}0.00640 * * * \\
\left(<1 \times 10^{-12}\right)\end{array}$ & $\begin{array}{l}0.00711^{* * * *} \\
\left(<1 \times 10^{-12}\right)\end{array}$ & $\begin{array}{l}0.00835 * * * \\
\left(<1 \times 10^{-12}\right)\end{array}$ & $\begin{array}{l}0.00889 * * * \\
\left(<1 \times 10^{-12}\right)\end{array}$ \\
\hline$T U R N_{i t}$ & $\begin{array}{c}2.70 \times 10^{-5} \\
(0.236)\end{array}$ & $\begin{array}{c}2.70 \times 10^{-5} \\
(0.236)\end{array}$ & $\begin{array}{c}3.56 \times 10^{-5} \\
(0.118)\end{array}$ & $\begin{array}{c}1.19 \times 10^{-5} \\
(0.643)\end{array}$ & $\begin{array}{c}2.80 \times 10^{-5} \\
(0.275)\end{array}$ & $\begin{array}{c}0.000197 \\
(0.405)\end{array}$ \\
\hline$T U R N_{i t-1}$ & & & & & & $\begin{array}{c}7.35 \times 10^{-5} \\
(0.756)\end{array}$ \\
\hline$R_{m t}-R_{f t}$ & $\begin{array}{c}1.022^{* * * *} \\
\left(<1 \times 10^{-12}\right)\end{array}$ & $\begin{array}{c}1.022 * * * \\
\left(<1 \times 10^{-12}\right)\end{array}$ & $\begin{array}{c}0.986 * * * \\
\left(<1 \times 10^{-12}\right)\end{array}$ & $\begin{array}{c}1.031^{* * * *} \\
\left(<1 \times 10^{-12}\right)\end{array}$ & $\begin{array}{c}0.997 * * * \\
\left(<1 \times 10^{-12}\right)\end{array}$ & $\begin{array}{c}0.972 * * * \\
\left(<1 \times 10^{-12}\right)\end{array}$ \\
\hline$S M B_{t}$ & $\begin{array}{c}0.532^{* * * *} \\
\left(<1 \times 10^{-12}\right)\end{array}$ & $\begin{array}{c}0.532 * * * \\
\left(<1 \times 10^{-12}\right)\end{array}$ & $\begin{array}{c}0.496^{* * *} \\
\left(<1 \times 10^{-12}\right)\end{array}$ & $\begin{array}{c}0.503^{* * * *} \\
\left(<1 \times 10^{-12}\right)\end{array}$ & $\begin{array}{c}0.467 * * * \\
\left(<1 \times 10^{-12}\right)\end{array}$ & $\begin{array}{c}0.456^{* * * *} \\
\left(<1 \times 10^{-12}\right)\end{array}$ \\
\hline$H M L_{t}$ & $\begin{array}{l}0.0574 * * * \\
\left(<1 \times 10^{-12}\right)\end{array}$ & $\begin{array}{l}0.0574 * * * \\
\left(<1 \times 10^{-12}\right)\end{array}$ & $\begin{array}{l}0.0571^{* * * *} \\
\left(<1 \times 10^{-12}\right)\end{array}$ & $\begin{array}{l}0.0557^{* * * *} \\
\left(<1 \times 10^{-12}\right)\end{array}$ & $\begin{array}{l}0.0541^{* * * *} \\
\left(<1 \times 10^{-12}\right)\end{array}$ & $\begin{array}{l}0.0732^{* * *} \\
\left(<1 \times 10^{-12}\right)\end{array}$ \\
\hline$E X P_{i t}$ & & $\begin{array}{c}-0.000786 \\
(0.567)\end{array}$ & & & $\begin{array}{c}-0.000892 \\
(0.559)\end{array}$ & $\begin{array}{c}0.000499 \\
(0.851)\end{array}$ \\
\hline$E X P_{i t-1}$ & & & & & & $\begin{array}{c}-0.00213 \\
(0.514)\end{array}$ \\
\hline$M I C R O_{t}$ & & & $\begin{array}{l}0.0307 * * * \\
\left(<1 \times 10^{-12}\right)\end{array}$ & & $\begin{array}{c}0.0309^{* * *} \\
\left(<1 \times 10^{-12}\right)\end{array}$ & $\begin{array}{l}0.0449^{* * * *} \\
\left(<1 \times 10^{-12}\right)\end{array}$ \\
\hline$M_{G R Y R S_{i t}}$ & & & & $\begin{array}{c}-0.000292 * * \\
(0.0231)\end{array}$ & $\begin{array}{c}-0.000288^{* *} \\
(0.0248)\end{array}$ & $\begin{array}{c}0.000267 * * \\
(0.0464)\end{array}$ \\
\hline Obs. & 43851 & 43851 & 43851 & 29040 & 29040 & 27037 \\
\hline$R^{2}$ & 0.771 & 0.771 & 0.772 & 0.718 & 0.719 & 0.710 \\
\hline \multicolumn{7}{|c|}{ Panel D: Micro Cap (116 Funds) } \\
\hline & $(1)$ & $(2)$ & (3) & (4) & $(5)$ & $(6)$ \\
\hline$\alpha_{i}$ & $\begin{array}{c}0.00805 \\
(0.185)\end{array}$ & $\begin{array}{c}0.00292 \\
(0.677)\end{array}$ & $\begin{array}{c}0.00640 \\
(0.304)\end{array}$ & $\begin{array}{c}0.00499 \\
(0.770)\end{array}$ & $\begin{array}{l}0.0312 \\
(0.392)\end{array}$ & $\begin{array}{l}0.0140 \\
(0.777)\end{array}$ \\
\hline$T U R N_{i t}$ & $\begin{array}{c}0.0503^{* * *} \\
\left(3.53 \times 10^{-6}\right)\end{array}$ & $\begin{array}{c}0.0532 * * * \\
\left(5.43 \times 10^{-5}\right)\end{array}$ & $\begin{array}{c}-0.00359 \\
(0.614)\end{array}$ & $\begin{array}{c}0.0582^{* * *} \\
\left(7.34 \times 10^{-7}\right)\end{array}$ & $\begin{array}{l}0.0102 \\
(0.352)\end{array}$ & $\begin{array}{l}0.0139 \\
(0.530)\end{array}$ \\
\hline$T U R N_{i t-1}$ & & & & & & $\begin{array}{l}0.0189 \\
(0.548)\end{array}$ \\
\hline$R_{m t}-R_{f t}$ & $\begin{array}{c}1.092^{* * * *} \\
\left(<1 \times 10^{-12}\right)\end{array}$ & $\begin{array}{c}1.087 * * * \\
\left(<1 \times 10^{-12}\right)\end{array}$ & $\begin{array}{c}1.129 * * * \\
\left(<1 \times 10^{-12}\right)\end{array}$ & $\begin{array}{c}0.981 * * * \\
\left(<1 \times 10^{-12}\right)\end{array}$ & $\begin{array}{c}0.963 * * * \\
\left(<1 \times 10^{-12}\right)\end{array}$ & $\begin{array}{c}1.030^{* * *} \\
\left(2.06 \times 10^{-6}\right)\end{array}$ \\
\hline$S M B_{t}$ & $\begin{array}{c}0.844^{* * * *} \\
\left(<1 \times 10^{-12}\right)\end{array}$ & $\begin{array}{c}0.845^{* * * *} \\
\left(<1 \times 10^{-12}\right)\end{array}$ & $\begin{array}{c}0.883^{* * *} \\
\left(<1 \times 10^{-12}\right)\end{array}$ & $\begin{array}{c}1.010^{* * * *} \\
\left(1.75 \times 10^{-9}\right)\end{array}$ & $\begin{array}{c}0.957 * * * \\
\left(1.37 \times 10^{-6}\right)\end{array}$ & $\begin{array}{c}0.941^{* * *} \\
\left(3.24 \times 10^{-5}\right)\end{array}$ \\
\hline$H M L_{t}$ & $\begin{array}{c}-0.0569 \\
(0.227)\end{array}$ & $\begin{array}{c}-0.0571 \\
(0.224)\end{array}$ & $\begin{array}{c}-0.0455 \\
(0.343)\end{array}$ & $\begin{array}{c}-0.0204 \\
(0.834)\end{array}$ & $\begin{array}{c}-0.0432 \\
(0.687)\end{array}$ & $\begin{array}{c}-0.00894 \\
(0.944)\end{array}$ \\
\hline$E X P_{i t}$ & & $\begin{array}{l}-3.238 \\
(0.148)\end{array}$ & & & $\begin{array}{c}8.163 \\
(0.259)\end{array}$ & $\begin{array}{c}16.94 \\
(0.988)\end{array}$ \\
\hline$E X P_{i t-1}$ & & & & & & $\begin{array}{l}-13.08 \\
(0.991)\end{array}$ \\
\hline$M I C R O_{t}$ & & & $\begin{array}{l}0.0395 \\
(0.242)\end{array}$ & & $\begin{array}{l}0.0522 \\
(0.554)\end{array}$ & $\begin{array}{c}-0.00578 \\
(0.967)\end{array}$ \\
\hline$M G R Y R S_{i t}$ & & & & $\begin{array}{c}-0.00136 \\
(0.414)\end{array}$ & $\begin{array}{c}-0.000945 \\
(0.721)\end{array}$ & $\begin{array}{c}-0.000174 \\
(0.960)\end{array}$ \\
\hline Obs. & 2157 & 2157 & 2157 & 2105 & 2105 & 1035 \\
\hline$R^{2}$ & 0.921 & 0.922 & 0.922 & 0.914 & 0.918 & 0.923 \\
\hline
\end{tabular}

Note. Coefficient estimates for ordinary least squares regression of mutual fund quarterly excess net returns on several explanatory variables are presented across specifications (1) through (6). Corresponding $P$-values for the coefficient estimates are in parentheses. *, **, and $* * *$ indicate statistical significance at the $10 \%, 5 \%$, and $1 \%$ level, respectively. Variable abbreviations are described in the methods section. Number of observations (Obs.); coefficient of determination $\left(R^{2}\right)$.

Table 3 suggests that managers of mutual funds in all market cap focus categories are at least making the right trades on average. In the absence of fees, portfolio turnover is positively associated with excess return for every type of fund in nearly all of the specifications. The trend in loadings on $T U R N_{i t}$ is also similar to that in Table 2. Smaller cap funds still appear to benefit more from trading than larger cap funds. The strength of this finding is 
reinforced by specification (6) in each panel. All of the coefficients on the lagged turnover and expense ratio terms are not significant, which implies that returns in one period are minimally influenced by previous investment decisions and sunk costs, at least in the setting at hand. The counterintuitive inference from all the results so far is that trading confers more benefit when it involves stocks that are more difficult to trade. Yet a different method of measuring turnover impact portrays the opposite story, as the next subsection will show.

Table 3. Regression of mutual fund excess gross returns on turnover rate and other controls

\begin{tabular}{|c|c|c|c|c|c|c|}
\hline \multicolumn{7}{|c|}{ Panel A: Large Cap (2163 Funds) } \\
\hline & $(1)$ & $(2)$ & (3) & $(4)$ & $(5)$ & $(6)$ \\
\hline$\alpha_{i}$ & $\begin{array}{l}0.0800^{* * * *} \\
\left(<1 \times 10^{-12}\right)\end{array}$ & $\begin{array}{l}0.0838 * * * \\
\left(<1 \times 10^{-12}\right)\end{array}$ & $\begin{array}{c}0.0775^{* * *} \\
\left(<1 \times 10^{-12}\right)\end{array}$ & $\begin{array}{l}0.0760^{* * * *} \\
\left(<1 \times 10^{-12}\right)\end{array}$ & $\begin{array}{l}0.0772^{* * * *} \\
\left(<1 \times 10^{-12}\right)\end{array}$ & $\begin{array}{l}0.0770^{* * * *} \\
\left(<1 \times 10^{-12}\right)\end{array}$ \\
\hline$T U R N_{i t}$ & $\begin{array}{c}0.00208^{* *} \\
(0.0356)\end{array}$ & $\begin{array}{c}0.00227 * * \\
(0.0229)\end{array}$ & $\begin{array}{c}0.00187 * * * \\
(0.0592)\end{array}$ & $\begin{array}{c}0.00288 * * \\
(0.0171)\end{array}$ & $\begin{array}{c}0.00283 * * \\
(0.0201)\end{array}$ & $\begin{array}{c}0.00183 * \\
(0.0505)\end{array}$ \\
\hline$T U R N_{i t-1}$ & & & & & & $\begin{array}{c}-0.00116 \\
(0.354)\end{array}$ \\
\hline$R_{m t}-R_{f t}$ & $\begin{array}{c}0.959^{* * * *} \\
\left(<1 \times 10^{-12}\right)\end{array}$ & $\begin{array}{c}0.959 * * * \\
\left(<1 \times 10^{-12}\right)\end{array}$ & $\begin{array}{c}0.987 * * * \\
\left(<1 \times 10^{-12}\right)\end{array}$ & $\begin{array}{c}0.957 * * * \\
\left(<1 \times 10^{-12}\right)\end{array}$ & $\begin{array}{c}0.983^{* * *} \\
\left(<1 \times 10^{-12}\right)\end{array}$ & $\begin{array}{c}0.980^{* * * *} \\
\left(<1 \times 10^{-12}\right)\end{array}$ \\
\hline$S M B_{t}$ & $\begin{array}{l}-0.110^{* * *} \\
\left(<1 \times 10^{-12}\right)\end{array}$ & $\begin{array}{l}-0.111^{* * *} \\
\left(<1 \times 10^{-12}\right)\end{array}$ & $\begin{array}{l}-0.0774 * * * \\
\left(<1 \times 10^{-12}\right)\end{array}$ & $\begin{array}{l}-0.126^{* * *} \\
\left(<1 \times 10^{-12}\right)\end{array}$ & $\begin{array}{l}-0.0909^{* * *} \\
\left(<1 \times 10^{-12}\right)\end{array}$ & $\begin{array}{l}-0.0881^{* * *} \\
\left(<1 \times 10^{-12}\right)\end{array}$ \\
\hline$H M L_{t}$ & $\begin{array}{l}-0.0139^{* * *} \\
\left(1.83 \times 10^{-6}\right)\end{array}$ & $\begin{array}{l}-0.0136^{* * *} \\
\left(3.25 \times 10^{-6}\right)\end{array}$ & $\begin{array}{l}-0.0423^{* * *} \\
\left(<1 \times 10^{-12}\right)\end{array}$ & $\begin{array}{l}-0.0362^{* * *} \\
\left(<1 \times 10^{-12}\right)\end{array}$ & $\begin{array}{l}-0.0630^{* * *} \\
\left(<1 \times 10^{-12}\right)\end{array}$ & $\begin{array}{l}-0.0603^{* * *} \\
\left(<1 \times 10^{-12}\right)\end{array}$ \\
\hline$E X P_{i t}$ & & $\begin{array}{c}-0.832^{* * * *} \\
\left(4.97 \times 10^{-9}\right)\end{array}$ & & & $\begin{array}{c}-1.176^{* * *} \\
\left(3.90 \times 10^{-8}\right)\end{array}$ & $\begin{array}{l}-1.012 \\
(0.114)\end{array}$ \\
\hline$E X P_{i t-1}$ & & & & & & $\begin{array}{c}0.739 \\
(0.549)\end{array}$ \\
\hline$M I C R O_{t}$ & & & $\begin{array}{l}-0.0170^{* * * *} \\
\left(<1 \times 10^{-12}\right)\end{array}$ & & $\begin{array}{l}-0.0154^{* * *} \\
\left(1.54 \times 10^{-5}\right)\end{array}$ & $\begin{array}{c}-0.0134 * * * \\
(0.000256)\end{array}$ \\
\hline$M_{G R Y R S_{i t}}$ & & & & $\begin{array}{c}0.000597 \\
(0.178)\end{array}$ & $\begin{array}{c}0.000530 \\
(0.232)\end{array}$ & $\begin{array}{c}-0.000129 \\
(0.146)\end{array}$ \\
\hline Obs. & 16878 & 16878 & 16878 & 11790 & 11790 & 9053 \\
\hline$R^{2}$ & 0.871 & 0.871 & 0.907 & 0.870 & 0.904 & 0.905 \\
\hline \multicolumn{7}{|c|}{ Panel B: Mid Cap (2194 Funds) } \\
\hline & $(1)$ & (2) & (3) & $(4)$ & $(5)$ & $(6)$ \\
\hline$\alpha_{i}$ & $\begin{array}{c}0.117 * * * \\
\left(<1 \times 10^{-12}\right)\end{array}$ & $\begin{array}{c}0.114^{* * * *} \\
\left(<1 \times 10^{-12}\right)\end{array}$ & $\begin{array}{c}0.118^{* * * *} \\
\left(<1 \times 10^{-12}\right)\end{array}$ & $\begin{array}{c}0.120^{* * * *} \\
\left(<1 \times 10^{-12}\right)\end{array}$ & $\begin{array}{c}0.120 * * * \\
\left(<1 \times 10^{-12}\right)\end{array}$ & $\begin{array}{l}0.120 * * * \\
\quad\left(<1 \times 10^{-12}\right)\end{array}$ \\
\hline$T U R N_{i t}$ & $\begin{array}{c}0.000135 \\
(0.836)\end{array}$ & $\begin{array}{c}2.63 \times 10^{-5} \\
(0.968)\end{array}$ & $\begin{array}{c}4.94 \times 10^{-5} \\
(0.940)\end{array}$ & $\begin{array}{c}2.92 \times 10^{-5} \\
(0.966)\end{array}$ & $\begin{array}{c}-0.000176 \\
(0.803)\end{array}$ & $\begin{array}{c}-0.00375 \\
(0.812)\end{array}$ \\
\hline$T U R N_{i t-1}$ & & & & & & $\begin{array}{c}0.00299 \\
(0.423)\end{array}$ \\
\hline$R_{m t}-R_{f t}$ & $\begin{array}{c}1.056^{* * *} \\
\left(<1 \times 10^{-12}\right)\end{array}$ & $\begin{array}{c}1.056^{* * * *} \\
\left(<1 \times 10^{-12}\right)\end{array}$ & $\begin{array}{c}0.962 * * * \\
\left(<1 \times 10^{-12}\right)\end{array}$ & $\begin{array}{c}1.048^{* * * *} \\
\left(<1 \times 10^{-12}\right)\end{array}$ & $\begin{array}{c}0.958 * * * \\
\left(<1 \times 10^{-12}\right)\end{array}$ & $\begin{array}{c}0.962 * * * \\
\left(<1 \times 10^{-12}\right)\end{array}$ \\
\hline$S M B_{t}$ & $\begin{array}{c}0.197^{* * * *} \\
\left(<1 \times 10^{-12}\right)\end{array}$ & $\begin{array}{c}0.197 * * * \\
\left(<1 \times 10^{-12}\right)\end{array}$ & $\begin{array}{c}0.117 * * * \\
\left(<1 \times 10^{-12}\right)\end{array}$ & $\begin{array}{c}0.184 * * * \\
\left(<1 \times 10^{-12}\right)\end{array}$ & $\begin{array}{c}0.135 * * * \\
\left(<1 \times 10^{-12}\right)\end{array}$ & $\begin{array}{c}0.123 * * * \\
\left(<1 \times 10^{-12}\right)\end{array}$ \\
\hline$H M L_{t}$ & $\begin{array}{l}-0.0615^{* * *} \\
\left(<1 \times 10^{-12}\right)\end{array}$ & $\begin{array}{l}-0.0613^{* * *} \\
\left(<1 \times 10^{-12}\right)\end{array}$ & $\begin{array}{l}-0.0626^{* * *} \\
\left(<1 \times 10^{-12}\right)\end{array}$ & $\begin{array}{l}-0.0693^{* * *} \\
\left(<1 \times 10^{-12}\right)\end{array}$ & $\begin{array}{l}-0.0762^{* * *} \\
\left(<1 \times 10^{-12}\right)\end{array}$ & $\begin{array}{l}-0.0781^{* * *} \\
\left(<1 \times 10^{-12}\right)\end{array}$ \\
\hline$E X P_{i t}$ & & $\begin{array}{c}-0.712^{* * *} \\
\left(5.72 \times 10^{-5}\right)\end{array}$ & & & $\begin{array}{c}-1.048 * * * \\
\left(6.59 \times 10^{-6}\right)\end{array}$ & $\begin{array}{l}-0.592 \\
(0.319)\end{array}$ \\
\hline$E X P_{i t-1}$ & & & & & & $\begin{array}{c}0.337 \\
(0.573)\end{array}$ \\
\hline$M I C R O_{t}$ & & & $\begin{array}{c}-0.0190 \\
(0.320)\end{array}$ & & $\begin{array}{c}-0.0345 \\
(0.135)\end{array}$ & $\begin{array}{l}0.0554 * * * \\
\left(<1 \times 10^{-12}\right)\end{array}$ \\
\hline$M_{G R Y R S_{i t}}$ & & & & $\begin{array}{c}-0.000509 \\
(0.260)\end{array}$ & $\begin{array}{c}-0.000518 \\
(0.253)\end{array}$ & $\begin{array}{c}-0.000123 \\
(0.323)\end{array}$ \\
\hline Obs. & 17533 & 17533 & 17533 & 13151 & 13151 & 9492 \\
\hline$R^{2}$ & 0.821 & 0.821 & 0.877 & 0.817 & 0.882 & 0.881 \\
\hline
\end{tabular}




\begin{tabular}{|c|c|c|c|c|c|c|}
\hline \multicolumn{7}{|c|}{ Panel C: Small Cap (2255 Funds) } \\
\hline & $(1)$ & $(2)$ & (3) & (4) & $(5)$ & $(6)$ \\
\hline$\alpha_{i}$ & $\begin{array}{c}0.132 * * * \\
\left(<1 \times 10^{-12}\right)\end{array}$ & $\begin{array}{c}0.134 * * * \\
\left(<1 \times 10^{-12}\right)\end{array}$ & $\begin{array}{c}0.131^{* * *} \\
\left(<1 \times 10^{-12}\right)\end{array}$ & $\begin{array}{c}0.136^{* * *} \\
\left(<1 \times 10^{-12}\right)\end{array}$ & $\begin{array}{c}0.141^{* * *} \\
\left(<1 \times 10^{-12}\right)\end{array}$ & $\begin{array}{c}0.141^{* * *} \\
\left(<1 \times 10^{-12}\right)\end{array}$ \\
\hline$T_{U R N_{i t}}$ & $\begin{array}{c}0.00590 * * * \\
(0.00359)\end{array}$ & $\begin{array}{c}0.00598 * * * \\
(0.00324)\end{array}$ & $\begin{array}{c}0.00587 * * * \\
(0.00379)\end{array}$ & $\begin{array}{c}0.00503 * * \\
(0.0328)\end{array}$ & $\begin{array}{c}0.00522 * * \\
(0.0271)\end{array}$ & $\begin{array}{c}0.00531 * * \\
(0.0266)\end{array}$ \\
\hline$T U R N_{i t-1}$ & & & & & & $\begin{array}{c}0.000510 \\
(0.870)\end{array}$ \\
\hline$R_{m t}-R_{f t}$ & $\begin{array}{c}1.005^{* * *} \\
\left(<1 \times 10^{-12}\right)\end{array}$ & $\begin{array}{c}1.006^{* * *} \\
\left(<1 \times 10^{-12}\right)\end{array}$ & $\begin{array}{c}0.968^{* * * *} \\
\left(<1 \times 10^{-12}\right)\end{array}$ & $\begin{array}{c}0.992 * * * \\
\left(<1 \times 10^{-12}\right)\end{array}$ & $\begin{array}{c}0.964 * * * \\
\left(<1 \times 10^{-12}\right)\end{array}$ & $\begin{array}{c}0.966^{* * *} \\
\left(<1 \times 10^{-12}\right)\end{array}$ \\
\hline$S M B_{t}$ & $\begin{array}{c}0.551^{* * *} \\
\left(<1 \times 10^{-12}\right)\end{array}$ & $\begin{array}{c}0.551^{* * * *} \\
\left(<1 \times 10^{-12}\right)\end{array}$ & $\begin{array}{c}0.540^{* * * *} \\
\left(<1 \times 10^{-12}\right)\end{array}$ & $\begin{array}{c}0.558^{* * *} * \\
\left(<1 \times 10^{-12}\right)\end{array}$ & $\begin{array}{c}0.5433^{* * * *} \\
\left(<1 \times 10^{-12}\right)\end{array}$ & $\begin{array}{c}0.538 * * * \\
\left(<1 \times 10^{-12}\right)\end{array}$ \\
\hline$H M L_{t}$ & $\begin{array}{c}0.0849^{* * * *} \\
\left(<1 \times 10^{-12}\right)\end{array}$ & $\begin{array}{c}0.0850^{* * * *} \\
\left(<1 \times 10^{-12}\right)\end{array}$ & $\begin{array}{c}0.0384^{* * *} \\
\left(4.82 \times 10^{-9}\right)\end{array}$ & $\begin{array}{c}0.104 * * * \\
\left(<1 \times 10^{-12}\right)\end{array}$ & $\begin{array}{c}0.0543^{* * *} * \\
\left(2.77 \times 10^{-6}\right)\end{array}$ & $\begin{array}{c}0.0554 * * * \\
\left(5.94 \times 10^{-6}\right)\end{array}$ \\
\hline$E X P_{i t}$ & & $\begin{array}{l}-0.444 * \\
(0.0686)\end{array}$ & & & $\begin{array}{c}-0.848^{* *} \\
(0.0457)\end{array}$ & $\begin{array}{c}-0.848^{* *} \\
(0.0126)\end{array}$ \\
\hline$E X P_{i t-1}$ & & & & & & $\begin{array}{c}0.637 \\
(0.207)\end{array}$ \\
\hline$M I C R O_{t}$ & & & $\begin{array}{c}0.0364 * * * \\
\left(<1 \times 10^{-12}\right)\end{array}$ & & $\begin{array}{c}0.0312^{* * * *} \\
\left(2.89 \times 10^{-5}\right)\end{array}$ & $\begin{array}{l}0.0295^{* * *} \\
(0.000185)\end{array}$ \\
\hline$M_{G R Y R S_{i t}}$ & & & & $\begin{array}{c}-0.000409 \\
(0.459)\end{array}$ & $\begin{array}{c}-0.000443 \\
(0.423)\end{array}$ & $\begin{array}{c}-0.000131 \\
(0.517)\end{array}$ \\
\hline Obs. & 18405 & 18405 & 18405 & 13987 & 13987 & 12568 \\
\hline$R^{2}$ & 0.715 & 0.715 & 0.750 & 0.645 & 0.655 & 0.641 \\
\hline \multicolumn{7}{|c|}{ Panel D: Micro Cap (15 Funds) } \\
\hline & $(1)$ & $(2)$ & (3) & $(4)$ & $(5)$ & $(6)$ \\
\hline$\alpha_{i}$ & $\begin{array}{l}0.0709 * \\
(0.0923)\end{array}$ & $\begin{array}{l}0.0556 \\
(0.235)\end{array}$ & $\begin{array}{l}0.0736 \\
(0.253)\end{array}$ & $\begin{array}{c}0.184 \\
(0.170)\end{array}$ & $\begin{array}{c}0.129 \\
(0.374)\end{array}$ & $\begin{array}{c}0.203 \\
(0.143)\end{array}$ \\
\hline$T_{U R N} N_{i t}$ & $\begin{array}{c}0.0622 * * \\
(0.0446)\end{array}$ & $\begin{array}{c}0.00581 \\
(0.912)\end{array}$ & $\begin{array}{l}0.0626 * \\
(0.0879)\end{array}$ & $\begin{array}{l}0.0250 \\
(0.668)\end{array}$ & $\begin{array}{c}-0.0482 \\
(0.589)\end{array}$ & $\begin{array}{l}0.0359 \\
(0.140)\end{array}$ \\
\hline$T U R N_{i t-1}$ & & & & & & $\begin{array}{c}-0.0529 \\
(0.328)\end{array}$ \\
\hline$R_{m t}-R_{f t}$ & $\begin{array}{c}1.144 * * * \\
\left(<1 \times 10^{-12}\right)\end{array}$ & $\begin{array}{c}1.142^{* * * *} \\
\left(<1 \times 10^{-12}\right)\end{array}$ & $\begin{array}{c}1.256^{* * *} \\
\left(<1 \times 10^{-12}\right)\end{array}$ & $\begin{array}{c}1.222 * * * \\
\left(<1 \times 10^{-12}\right)\end{array}$ & $\begin{array}{c}1.257 * * * \\
\left(<1 \times 10^{-12}\right)\end{array}$ & $\begin{array}{c}1.254 * * * \\
\left(<1 \times 10^{-12}\right)\end{array}$ \\
\hline$S M B_{t}$ & $\begin{array}{c}0.901^{* * *} * \\
\left(<1 \times 10^{-12}\right)\end{array}$ & $\begin{array}{c}0.903^{* * * *} \\
\left(<1 \times 10^{-12}\right)\end{array}$ & $\begin{array}{c}0.952^{* * * *} \\
\left(<1 \times 10^{-12}\right)\end{array}$ & $\begin{array}{c}1.261^{* * *} * \\
\left(<1 \times 10^{-12}\right)\end{array}$ & $\begin{array}{c}1.095^{* * *} \\
\left(<1 \times 10^{-12}\right)\end{array}$ & $\begin{array}{c}1.075^{* * *} \\
\left(<1 \times 10^{-12}\right)\end{array}$ \\
\hline$H M L_{t}$ & $\begin{array}{c}-0.0976 \\
(0.149)\end{array}$ & $\begin{array}{c}-0.0983 \\
(0.148)\end{array}$ & $\begin{array}{c}-0.251^{* * *} \\
\left(2.31 \times 10^{-5}\right)\end{array}$ & $\begin{array}{c}-0.460^{* * *} \\
\left(9.61 \times 10^{-7}\right)\end{array}$ & $\begin{array}{l}-0.237 * * * \\
(0.000428)\end{array}$ & $\begin{array}{l}-0.243 * * * \\
(0.000718)\end{array}$ \\
\hline$E X P_{i t}$ & & $\begin{array}{l}-1.532 \\
(0.698)\end{array}$ & & & $\begin{array}{l}-3.432 \\
(0.393)\end{array}$ & $\begin{array}{l}-11.22 \\
(0.461)\end{array}$ \\
\hline$E X P_{i t-1}$ & & & & & & $\begin{array}{c}10.22 \\
(0.500)\end{array}$ \\
\hline$M I C R O_{t}$ & & & $\begin{array}{c}0.0491 * * \\
(0.0121)\end{array}$ & & $\begin{array}{c}0.0369 * * \\
(0.0130)\end{array}$ & $\begin{array}{l}0.102 * * \\
(0.0198)\end{array}$ \\
\hline$M_{G R Y R S_{i t}}$ & & & & $\begin{array}{c}-0.0118^{*} \\
(0.0690)\end{array}$ & $\begin{array}{c}-0.00664 \\
(0.401)\end{array}$ & $\begin{array}{c}0.00120 \\
(0.423)\end{array}$ \\
\hline Obs. & 88 & 88 & 88 & 38 & 38 & 33 \\
\hline$R^{2}$ & 0.041 & 0.065 & 0.041 & 0.187 & 0.217 & 0.243 \\
\hline
\end{tabular}

Note. Coefficient estimates for ordinary least squares regression of mutual fund quarterly excess gross returns on several explanatory variables are presented across specifications (1) through (6). Corresponding $P$-values for the coefficient estimates are in parentheses. *,**, and $* * *$ indicate statistical significance at the $10 \%, 5 \%$, and $1 \%$ level, respectively. Variable abbreviations are described in the methods section. There is a discrepancy between the number of funds listed in this table and the number of funds listed in Table 2 because fund gross returns were constructed from holdings data. Absent observations here are due to the unavailability of some individual stock returns. Number of observations (Obs.); coefficient of determination $\left(R^{2}\right)$. 


\subsection{Total Annual Impact of Turnover on Fund Performance}

The regressions in Tables 2 and 3 measure the incremental impact of portfolio turnover on mutual fund returns. Table 4 reports the own-benchmark abnormal return, the difference in return between trading and not trading for an entire year. Adding expense ratio back into the gross return allows the own-benchmark abnormal return to purely reflect the effects of trading, both the quality of trades and associated costs. Since Table 3 already demonstrated that the average fund manager investing in each market cap focus category is overall making good trade decisions, these negative abnormal returns with overwhelming statistical significance suggest that transaction costs are what cause excessive portfolio turnover to be undesirable. The return gap calculated here for micro cap funds is consistent with the findings of Kacperczyk, Sialm, and Zheng (2008).

Table 4. Summary of average own-benchmark abnormal returns

\begin{tabular}{lccccccc}
\hline & Mean & $t$-statistic & 25th \%tile & Median & 75th \%tile & Min. & Max. \\
\hline Large Cap Funds & -0.0698 & -58.68 & -0.1597 & -0.0732 & 0.0110 & -1.8594 & 0.9859 \\
Mid Cap Funds & -0.0885 & -74.41 & -0.1823 & -0.0847 & 0.0118 & -2.5321 & 1.0500 \\
Small Cap Funds & -0.1051 & -79.62 & -0.2106 & -0.1006 & 0.0104 & -3.1709 & 1.0519 \\
Micro Cap Funds & -0.1108 & -5.63 & -0.2622 & -0.1451 & 0.0264 & -3.5178 & 1.3420 \\
\hline
\end{tabular}

Note. For every fund at the end of every year, the one-year future gross return of the previous year's portfolio less the annual expense ratio was subtracted from the current annual net return. This difference was averaged across all observations within each mutual fund category. The $t$-statistics of average differences relative to zero are included.

\section{Discussion}

There is a glaring conflict in interpretation between the marginal impacts of portfolio turnover in Table 2 and portfolio own-benchmark abnormal returns in Table 4. Table 2 shows that with respect to net returns, additional trading is helpful to small cap and micro cap focused funds, but harmful to large cap and mid cap focused funds. In contrast, Table 4 indicates that trading hurts all four types of mutual funds on average. It is important to note that calculations for both tables take into account the costs of trading. However, Table 4's findings are more credible because they were derived from an experiment with simulated controls: the projected returns of previous portfolios.

Furthermore, the coefficient estimate on $T U R N_{i t}$ warrants careful examination. Turnover is measured as a fraction of total fund market value. As shown in Table 1, average market value and average shares are spread over a large range among the four types of funds. The average micro cap fund holds greater market value and more shares than its large cap, mid cap, and small cap counterparts. Therefore the same increment of turnover rate does not represent the same absolute volume of trading for the different types of funds. At the average, "a unit" of turnover represents greater trading volume for micro cap funds. Since transaction costs are based on total trading, not portfolio fraction, micro cap funds pay more "per unit" of turnover. They should thus have a more negative own-benchmark abnormal return in the context of approximately equal turnover rates across all funds, which Table 1 confirms.

Table 3 does not raise any disagreement. If a manager can trade without cost and equally intelligibly across all funds, and smaller cap stocks yield greater returns as they are supposed to based on risk, $T U R N_{i t}$ 's coefficient estimate must be greater for smaller cap funds.

\subsection{The Manager Effect and a Misleading Sign-Change}

An omitted variable bias was hypothesized to be responsible for the inconsistencies described above. Jin and Scherbina (2011) found that regardless of other characteristic differences, new managers sell off inherited losers at higher rates than stocks in any other momentum decile. Therefore the marginal impact of turnover should not be uniform. Instead, it should change depending on how long a particular manager has worked at the same fund.

A new series of regressions was constructed, again using net returns, but now including the term TURN $N_{i t}$ interacted with MGRYRS $S_{i t}$ EXP ${ }_{i t}$ and MICRO $O_{t}$ were omitted for parsimony, as their influences on TURN $N_{i t}$ 's coefficient estimate have been unimpressive. Table 5 presents the regression results for:

$$
\begin{aligned}
\left(R_{i t}-R_{f t}\right)= & \alpha_{i}+\tau_{i} \cdot T_{U R N_{i t}}+\zeta_{i} \cdot M_{G R Y R S_{i t}}+\varphi_{i} \cdot \text { TURN }_{i t} \cdot M \text { MRYRS }_{i t} \\
& +\beta_{i} \cdot\left(R_{m t}-R_{f t}\right)+s_{i} \cdot S M B_{t}+h_{i} \cdot H M L_{t}+\epsilon_{i t}
\end{aligned}
$$


Marginal impact of $T U R N_{i t}$ becomes $\frac{\partial\left(R_{i t}-R_{f t}\right)}{\partial\left(T U R N_{i t}\right)}=\tau_{i}+\varphi_{i} \cdot M G R Y R S_{i t}$, instead of $\frac{\partial\left(R_{i t}-R_{f f}\right)}{\partial\left(T U R N_{i t}\right)}=\tau_{i}$ as earlier.

Table 5. Regression of mutual fund excess net returns on turnover and a turner-manager interactive term

\begin{tabular}{lcccc}
\hline & Large Cap Funds & Mid Cap Funds & Small Cap Funds & Micro Cap Funds \\
\hline \multirow{2}{*}{$\alpha_{i}$} & $0.00331^{* * *}$ & 0.00133 & $0.00398^{* * *}$ & -0.0178 \\
& $(0.000134)$ & $(0.329)$ & $(0.00914)$ & $(0.468)$ \\
$T U R N_{i t}$ & 0.000246 & $0.00191^{*}$ & $0.00450^{* * *}$ & $0.0373^{* *}$ \\
& $(0.742)$ & $(0.0727)$ & $(0.000305)$ & $(0.0157)$ \\
MGRYRS $i t$ & $9.00 \times 10^{-5}$ & 0.000141 & 0.000430 & 0.00750 \\
& $(0.564)$ & $(0.584)$ & $(0.146)$ & $(0.261)$ \\
$T U R N_{i t} \cdot$ MGRYRS $_{i t}$ & -0.000130 & -0.000175 & $-0.000472^{*}$ & -0.00603 \\
& $(0.308)$ & $(0.392)$ & $(0.0682)$ & $(0.381)$ \\
$R_{m t}-R_{f t}$ & $0.957^{* * *}$ & $0.992^{* * *}$ & $1.048^{* * *}$ & $1.224^{* * *}$ \\
& $\left(<1 \times 10^{-12}\right)$ & $\left(<1 \times 10^{-12}\right)$ & $\left(<1 \times 10^{-12}\right)$ & $\left(<1 \times 10^{-12}\right)$ \\
$S M B_{t}$ & $-0.126^{* * *}$ & $0.185^{* * *}$ & $0.557^{* * *}$ & $1.259^{* * *}$ \\
& $\left(<1 \times 10^{-12}\right)$ & $\left(<1 \times 10^{-12}\right)$ & $\left(<1 \times 10^{-12}\right)$ & $\left(<1 \times 10^{-12}\right)$ \\
$H M L_{t}$ & $-0.0363^{* * *}$ & $-0.0692^{* * *}$ & $0.104^{* * *}$ & $-0.472^{* * *}$ \\
Obs. & $\left(<1 \times 10^{-12}\right)$ & $\left(<1 \times 10^{-12}\right)$ & $\left(<1 \times 10^{-12}\right)$ & $\left(7.33 \times 10^{-7}\right)$ \\
$R^{2}$ & 22691 & 24734 & 29040 & 2083 \\
\hline
\end{tabular}

Note. Coefficient estimates for ordinary least squares regression of mutual fund quarterly excess net returns on several explanatory variables are presented. Corresponding $P$-values for the coefficient estimates are in parentheses. *, **, and $* * *$ indicate statistical significance at the $10 \%, 5 \%$, and $1 \%$ level, respectively. Variable abbreviations are described in the methods section. Number of observations (Obs.); coefficient of determination $\left(R^{2}\right)$.

For every category of mutual funds, $\tau_{i}$ is positive and $\varphi_{i}$ is negative. This supports the findings of both Table 3 and Jin and Scherbina (2011). The pure impact of portfolio turnover is positive, which implies that managers are fulfilling their job responsibilities. However as $M G R Y R S_{i t}$ increases, the quality of trading decisions declines; possibly because they hold on to losers to a greater extent until a new manager eventually sells them off. It is also interesting to remark that the non-interacted $M G R Y R S_{i t}$ term has a positive coefficient in each regression. Even though it hardly carries any explanatory power, the coefficient sign hints that manager experience may positively contribute to fund returns, perhaps through means such as leadership and organization but less likely through executing trades with time.

Omission of an interaction term between $T U R N_{i t}$ and MGRYRS $S_{i t}$ from the regressions in Table 2 imposed a negative bias on the included variables' coefficients. The magnitude of this bias is different for the different fund focus categories. The average tenure of micro cap fund managers is about two years less than that of managers working at funds in the other focus categories. Therefore the downward bias on $T U R N_{i t}$ 's coefficient estimate in Panel D of Table 2 should be weaker than the downward bias in any of the other panels. Since the coefficient estimates on $T U R N_{i t}$ for large cap and mid cap funds are the smallest in the cost-free regressions of Table 3 , incorporation of downward bias in Table 2 actually turned those small positive coefficients into negative coefficients. The loading on $T U R N_{i t}$ stood as positive for small cap and micro cap funds.

An area for improvement is securing more complete fund holdings data so that gross returns are computable for every fund with available net returns data. The turnover-return relationship may then be assessed for other meaningful stratifications of portfolio holdings.

\subsection{Conclusion}

In summary, results from the seemingly innocuous regressions in Table 2 do not reflect a qualitatively different impact of turnover on the returns of different market cap focused mutual funds. Trading activity, by itself without fees, is on average beneficial for all of the fund focus categories addressed in this study. However, negative omitted-variable bias from the exclusion of a trading-manager interactive term inverts the sign of turnover's marginal impact for some fund focus categories but not others. Investment fund leadership may find utility in analyzing the historical turnover-return and manager-return trends for their own holdings of varying market capitalization to more effectively plan future strategies. 


\section{Acknowledgments}

This study was supported by an internal research grant from Sichuan College of Architectural Technology. Special thanks to Professor Shifu Deng and Professor Changzhou Qian for their help with the final drafting of this manuscript.

\section{References}

Barber, B., \& Odean, T. (2000). Trading is Hazardous to Your Wealth: The Common Stock Investment Performance of Individual Investors. Journal of Finance, 55, 773-806. https://doi.org/10.1111/0022-1082.00226

Carhart, M. M. (1997). On persistence in mutual fund performance. Journal of Finance, 52, 57-82. https://doi.org/10.1111/j.1540-6261.1997.tb03808.x

Chevalier, J., \& Ellison, G. (1997). Risk Taking by Mutual Funds as a Response to Incentives. Journal of Political Economy, 105, 1167-1200. https://doi.org/10.1086/516389

Glosten, L. R., \& Milgrom, P. R. (1985). Bid, ask, and transaction prices in a specialist market with heterogeneously informed traders. Journal of Financial Economics, 14, 71-100. https://doi.org/10.1016/0304-405X(85)90044-3

Jin, L., \& Scherbina, A. (2011). Inheriting Losers. Review of Financial Studies, 24, 786-820. https://doi.org/10.1093/rfs/hhq084

Kacperczyk, M., Sialm, C., \& Zheng, L. (2008). The Unobserved Actions of Mutual Funds. Review of Financial Studies, 21, 2379-2416. https://doi.org/10.1093/rfs/hhl041

Sadka, R., \& Scherbina, A. (2007). Analyst Disagreement, Mispricing, and Liquidity. Journal of Finance, 62, 2367-2403. https://doi.org/10.1111/j.1540-6261.2007.01278.x

\section{Copyrights}

Copyright for this article is retained by the author(s), with first publication rights granted to the journal.

This is an open-access article distributed under the terms and conditions of the Creative Commons Attribution license (http://creativecommons.org/licenses/by/4.0/). 